\title{
Experimental Determination of a Betatron Difference Resonance *
}

\author{
M. Ellison ${ }^{a}$, M. Ball ${ }^{a}$, B. Brabson ${ }^{a}$, J. Budnick ${ }^{a}$, D.D. Caussyn ${ }^{a}$, A. W. Chao ${ }^{b}, J$ Collins $^{a}$, \\ V. Derenchuk ${ }^{a}$, S. Dutt ${ }^{b}$, G. East ${ }^{a}$, T. Ellison ${ }^{a}$, D. Friesel ${ }^{a}$, B. Hamilton ${ }^{a}$, H. Huang ${ }^{a}$, \\ W.P. Jones ${ }^{a}$, S.Y. Lee ${ }^{a}$, D. $\mathrm{Li}^{a}$, M. Minty ${ }^{c}$, S. Nagaitsev ${ }^{a}$, K. Y. Ng ${ }^{d}$, X. Pei ${ }^{f}$, \\ T. Sloan ${ }^{a}$, M. Syphers ${ }^{b}$, L. Teng ${ }^{e}$, Y. Wang ${ }^{a}$, Y. T. Yan ${ }^{b}$, P.L. Zhang
}

\section{Abstract}

The betatron difference resonance, $Q_{z}-2 Q_{z}=-6$, where $Q_{z, z}$ are the number of betatron oscillations per turn, was studied at the Indiana University Cyclotron $\mathrm{Fa}$ cility (IUCF) cooler ring. The position of the beam was measured in both the horizontal and vertical planes of oscillation after a pulsed kicker magnet was fired to produce coherent motion. The effect of the coupling resonance was clearly observed and it was found that the subsequent particle motion could be described by a simple Hamiltonian. The resonance strength and tune shift as a function of betatron amplitude were measured.

\section{Introduction}

For particle motion in a circular accelerator, the betatron positions $x(s)$ and $z(s)$, around the closed orbit, in the presence of magnetic field errors $\Delta B_{x, z}$ are given by Hill's equation [1]:

$$
\frac{d^{2} x}{d s^{2}}+K_{z}(s) x=\frac{\Delta B_{z}}{B \rho} ; \frac{d^{2} z}{d s^{2}}+K_{z}(s) z=-\frac{\Delta B_{z}}{B \rho},
$$

with

$$
\Delta B_{z}+i \Delta B_{z}=B_{0} \sum_{n \geq 2}\left(b_{n}+i a_{n}\right)(z+i z)^{n}
$$

where $b_{n}$ and $a_{n}$ are the normal and the skew multipole components, respectively. The $K_{z, z}(s)$ are functions of the quadrupole strength, $B \rho=p c / e$ is the magnetic rigidity, and $s$ is the longitudinal particle coordinate which advances from 0 to $C$, the circumference, as the particle completes one revolution. Both $K_{z, x}(s)$ and the field error term $\Delta B_{x, x} / B \rho$ are periodic functions of $s$ with period $C$. The field error term $\Delta B_{z, z} / B \rho$, which arises from higherorder multipoles, is normally small. However when the resonance condition, $m Q_{z}+n Q_{z}=l$, with $m, n, l$ integers, is approached, particles will encounter coherent kicks from the multipoles. This coherent perturbation can have a profound effect on particle motion.

Based on the theory of betatron motion, the betatron amplitude of particle motion is given by, $x, z=$ $\sqrt{2 \beta_{z, z} J_{z, z}} \cos \left(\phi_{z, z}\right)$, where $J_{z, z}=\left(x^{2}, z^{2}+p_{x, z}^{2}\right) / 2 \beta_{x, z}$

*Work supported in part by a grant from NSF PHY-9221402 and from the U.S.DoE; a IUCF, Indiana University, Bloomington, IN 47405; ' The SSC laboratory, 2550 Beckleymeade Avenue, Dallas, TX 75237-3946; c SLAC, MS26, Box 4349, Stanford, CA 94308; d Fermilab, P.O. Box 500, Batavia, IL 60510; 'Argonne National Laboratory, APS, 9700 S. Cass Ave., IL 60439; $f$ Brookhoven Notional Laboratory, Upton, NY 11973. are the actions, $\beta$ is the beta function and $\phi$ is the betatron phase advance. Assuming a single resonance, the Hamiltonian can be written as [2],

$$
H \approx H_{o}\left(J_{z}, J_{z}\right)+g J_{z}^{\frac{|m|}{2}} J_{z}^{\frac{|m|}{2}} \cos \left(m \phi_{z}+n \phi_{z}-l \theta+\chi\right)
$$

where $g$ and $\chi$ are determined by the nonlinear multipoles which drive the resonance.

$$
H_{0}=Q_{z o} J_{z}+Q_{z o} J_{z}+\frac{\alpha_{z x}}{2} J_{z}^{2}+\alpha_{z z} J_{z} J_{z}+\frac{\alpha_{z z}}{2} J_{z}^{2}
$$

$\theta$ is the azimuthal angle, and $\alpha_{i j}=\partial Q_{i} / \partial J_{j}$. This Hamiltonian can be integrated after performing a canonical transformation with the generating function,

$$
F_{2}\left(\phi_{z}, \phi_{z}, J_{1}, J_{2}\right)=J_{1}\left(m \phi_{z}+n \phi_{z}-l \theta+\chi\right)+J_{2} \phi_{z} \text {. }
$$

The new coordinates are, $\phi_{1}=\left(m \phi_{z}+n \phi_{z}-l \theta+\chi\right)$, $\phi_{3}=\phi_{z}, J_{1}=J_{z} / m$, and $J_{2}=J_{z}-n J_{z} / m$. The new Hamiltonian, $\tilde{H}$, is given by,

$$
\begin{aligned}
\tilde{H} & =\delta_{1} J_{1}+\frac{\alpha_{11}}{2} J_{1}^{2}+\left[Q_{x \sigma} J_{2}+\frac{\alpha_{x z}}{2} J_{2}^{2}\right] \\
& +g\left(m J_{1}\right)^{\frac{|m|}{2}}\left(n J_{1}+J_{2}\right)^{\frac{|n|}{2}} \cos \left(\phi_{1}\right)
\end{aligned}
$$

where $\delta_{1}=m Q_{x 0}+n Q_{x 0}-l+\left(m \alpha_{z z}+n \alpha_{z z}\right) J_{2}$ and $\alpha_{11}=\alpha_{z x}+2 n \alpha_{z x}+n^{2} \alpha_{z x}$. This new Hamiltonian is independent of $\theta$ and $\phi_{2}$, thus $\vec{H}$ and $J_{2}$ are constants of motion which determine the particle motion completely.

If there is only a horizontal kick, $\left(J_{z o}=0\right)$ then for the resonance considered here where $m=1$ and $n=-2$ Eq. 4 becomes,

$$
\left(2 J_{1}-J_{2}\right)\left[\frac{\alpha_{11}}{4} J_{1}-g \sqrt{J_{1}} \cos \phi_{1}+\frac{\delta_{1}}{2}+\frac{\alpha_{11}}{8} J_{2}\right]=0
$$

The particle trajectory can be seen to follow the path of two intersecting circles in the map of $\left(\sqrt{J_{1}} \cos \phi_{1}, \sqrt{J_{1}} \sin \phi_{1}\right)$. The circle $2 J_{1}-J_{2}=0$ is called the "launching" circle, while the circle

$$
\frac{\alpha_{11}}{4} J_{1}-g \sqrt{J_{1}} \cos \phi_{1}+\frac{\delta_{1}}{2}+\frac{\alpha_{11}}{8} J_{2}=0
$$

is the nonlinear "coupling" circle. Matching the measured particle trajectories with the contours of constant $\bar{H}$ can determine $g, \alpha_{11}$ and $\delta_{1}$ only to an arbitrary multiplicative constant. If any one parameter is known then the "scale" is fixed and the other two parameters can also be uniquely determined.

\section{Experimental Method}




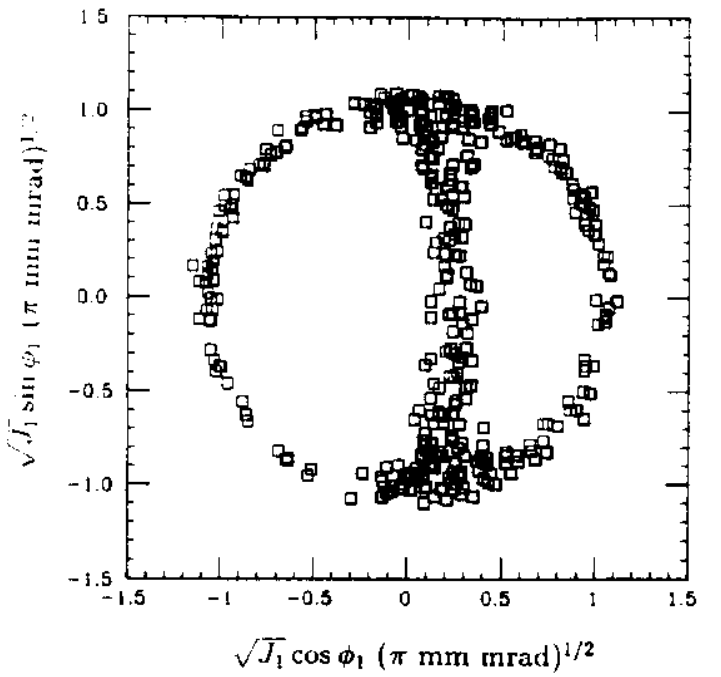

Figure 1: Plot of date obtained after a horizontal kick, dispiayed without averaging. The same kick is displayed in Fig. 2 with averaging (the dats set with the largest $J_{2}$ ).

Protons were injected into the IUCF cooler ring at 45 $\mathrm{MeV}$ on a ten second timing cycle. The beam was bunched at harmonic number 1 , with the rf cavity operating at $1.03168 \mathrm{MHz}$. The bunched beam was electron cooled for several seconds reducing its unnormalized rms emittance to $<0.3 \pi \mathrm{mm}$ mrad. The motion of a beam bunch with this small an emittance can closely simulate single particle motion. Since $J_{2}=J_{z}-n J_{z} / m$ is invariant, the difference resonance considered here is stable since the action in both planes of oscillation is bounded. Thus, it was possible to store beam with the betatron tunes on the resonance.

A coherent transverse oscillation was imparted to the bunch by firing puised kicker magnets. The subsequent motion was recorded on a turn-by-turn basis [3] for a complete grid of horizontal and vertical kicker strengths. The conversion from two position measurements $\left(\boldsymbol{x}_{1}, \boldsymbol{x}_{2}\right)$ in each plane to normalized position and momentum coordinates $\left(\boldsymbol{x}_{1}, \boldsymbol{p}_{\boldsymbol{x}}\right)$ is described elsewhere in these proceedings [4]. The gain of the data acquisition system was calibrated against the beam position monitoring system in the cooler ring which itself was calibrated against a wire scanner. The uncertainty in position gain is estimated to be $\pm 10 \%$.

\section{Data and Analysis}

\section{A. Data Reduction}

The two position measurements, in each plane of oscillation, were converted to normalized coordinates and both $\phi_{x, z}$, from which $\phi_{1}$ was derived, and $J_{z}$ were computed for each turn. So that the features of the nonlinear coupling resonance could be seen more clearly a 10-turn running average was used in all cases except for $\phi_{1}$ when only a horizontal kick was applied and no coherent motion had yet developed in the vertical plane. With no coherent motion the measurement of $\phi_{z}$ was not meaningful. An example of the data displayed without averaging is shown in Fig. 1.

It was thought that measurements of the tune shift with

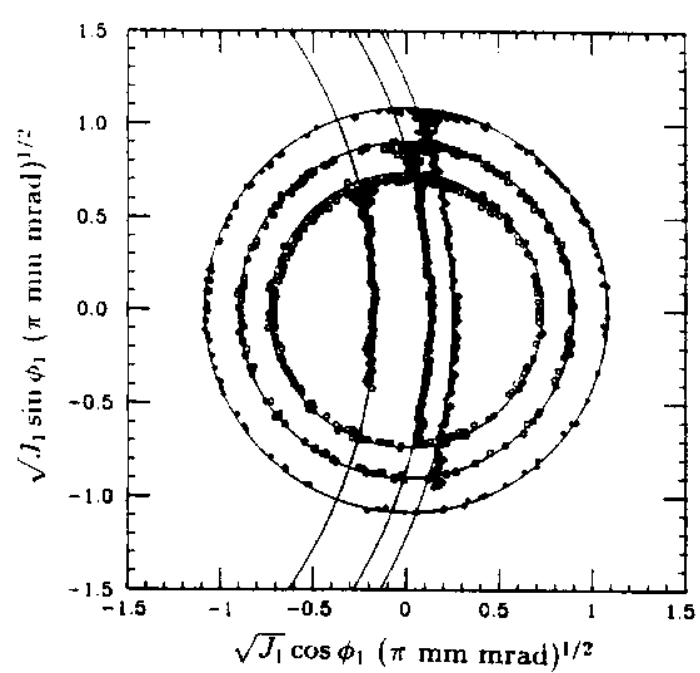

Figure 2: Plot of data (symbols) and fit (solid lines) to the Hamiltonian Eq. 5 for three different values of $J_{2}$. There was no vertical kick and both the leunching and coupling circies are shown.

amplitude parameters $\alpha_{z z}, \alpha_{z z}$ and $\alpha_{z z}$ could be made directly. With $\alpha_{11}$ known both $g$ and $\delta_{1}$ could then be determined. To measure the $\alpha$ parameters one need only find the slope of the measured tune verses $J$. However, our measurements were complicated by two factors. Firstly, the unperturbed tunes are not stable in the cooler ring. If one tracks the phase in $\left(x, z ; p_{a, x}\right)$ phase space it is possible to measure the tune, assuming a pure tone, in as few as $\mathbf{5 0}$ turns. Using this method of tracking the tune a $60 \mathrm{~Hz}$ modulation was observed in $Q_{x}$ of \pm 0.0005 and in $Q_{z}$ of \pm 0.002 . This uncertainty in the tune prior to the kick resulted in a large scatter of the measured tunes after the kick. Secondly, the resonance stop band was larger than anticipated so that even with $\left|Q_{z}-2 Q_{x}+6\right|>0.05$ the effect of the resonance was evident.

It was latter found that a two-kick procedure allows for the measurement of the $\alpha$ parameters even when the base tunes are not stable. The bunch is first given a small coherent oscillation by using the rf knock out system. This coherent motion allows the phase of the betation oscillation to be tracked and thus, the base tune to be measured. The bunch is then given a larger kick with one of the pulsed kicker magnets 500 turns later. In this way the tune change resulting from the change in $J$ can be accurately measured. The $\alpha$ parameters were measured using the two-kick proceedure when $Q_{z}-2 Q_{x}+6 \approx-0.08$. While the value of $\alpha_{11}$, determined at this tune location differed from the value of $\alpha_{11}$ determined from the resonance data the measurements will be useful when modeling the nonlinear elements in the cooler ring.

\section{B. Fitting the Measured Tune}

While the contours of constant $\tilde{H}$ are invariant if $g, \alpha_{11}$ and $\delta_{1}$ are each multiplied by the same constant, the phase advance per turn, $\Delta \phi_{1}$, is not invariant. By differentiating 


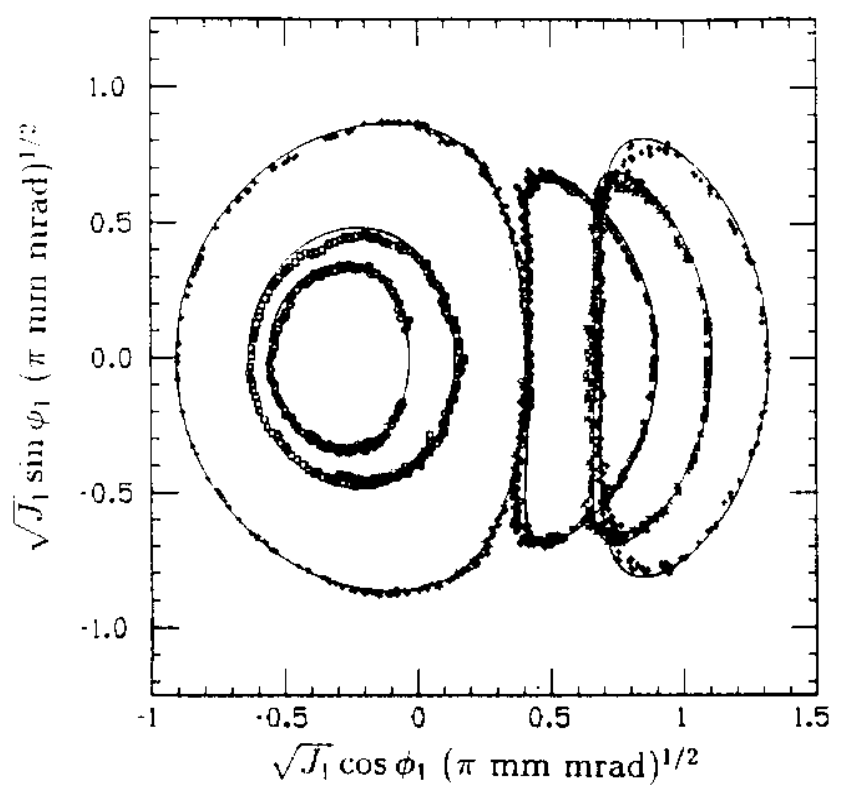

Figure 3: Plot of data (symbols) and fit (solid lines) to the Hamiltonian Eq. 4. The vertical kick was held constant and strength of the horieontal kick was varied.

the Hamiltonian one finds,

$$
\begin{aligned}
& \frac{\partial \tilde{H}}{\partial J_{1}}=\frac{d \phi_{1}}{d \theta} \approx \frac{\Delta \phi_{1}}{2 \pi} \\
& \frac{\partial \tilde{H}}{\partial J_{1}}=\delta_{1}+\alpha_{11} J_{1}+g \cos \phi_{1}\left(-3 J_{1}^{1 / 2}+\frac{J_{2}}{2 J_{1}^{1 / 2}}\right) .
\end{aligned}
$$

Using $\delta_{1}$ and $g$ as adjustable parameters with $\chi$ fixed at -1.01 radians, the measured phase advance of $\phi_{1}$ was fit to Eq. 6. Fitting 25 different kicks the mean resonance strength was $-493 \times 10^{-6}(\pi \mathrm{mm} \text { mrad })^{-1 / 2}$ with a standard deviation of $\pm 52 \times 10^{-6}$. Since the phase advance of $\phi_{1}$ was not very sensitive to the value of $\alpha_{11}$, the value of $\alpha_{11}$ was found instead by matching the curvature of the nonlinear coupling circle, when only a horizontal kick was applied, to the experimental data. The parameter $\alpha_{11}$ was found to be $350 \times 10^{-8}(\pi \mathrm{mm} \mathrm{mrad})^{-1}$ with the uncertainty estimated at $\pm 50 \times 10^{-6}$.

There was also a systematic variation in $\delta_{1}$ with $J_{2}$. From the slope of $\delta_{1}$ vs. $J_{2}$ the quantity $\alpha_{x z}-2 \alpha_{3 z}$ was found to be $(-160 \pm 25) \times 10^{-6}(\pi \mathrm{mm} \mathrm{mrad})^{-1}$. The values of $g, \alpha_{11}$ and $\chi$ were held constant when fitting the Hamiltonian to the experimental data.

\section{Fitting the Hamiltonian}

When only a horizontal kick was applied Eq. 5 was used to draw both the launching and coupling circles. The data was inspected to find $J_{2}=J_{x}+2 J_{z}$ and $\delta_{1}$ was adjusted for each kick. Three data sets with different values of $J_{2}$ are shown in Fig. 2. It was by fitting the curvature of the coupling circle to this data that the value of $\alpha_{11}$ was determined.

When there was both a horizontal and a vertical kick the data was fit to Eq. 4. The values of $g, \alpha_{11}$ and $\chi$ were again held constant, the value of $\delta_{1}$ was adjusted for each kick and the data was inspected to find the maximum of $J_{1}$.

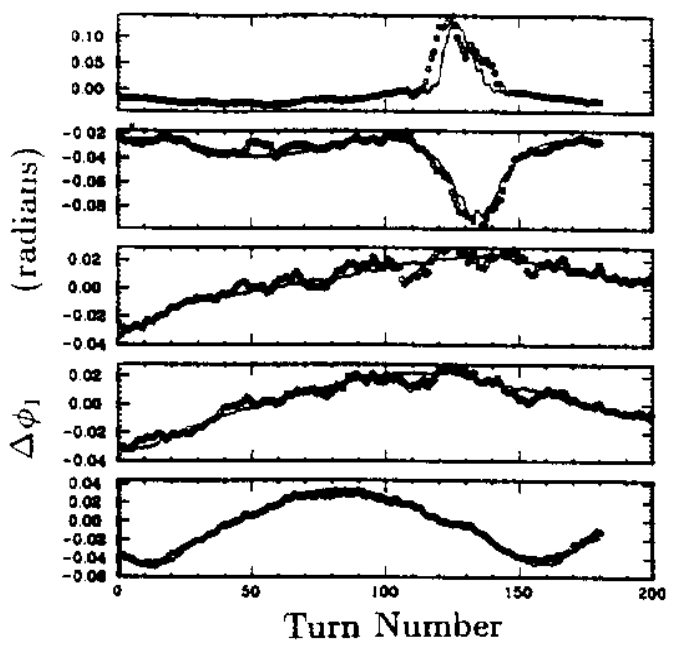

Figure 4: Plot of measured (symbols) and predicted (solid lines) tune for five of the six kicks shown in Fig. 3.

The value of $J_{2}$ used was the sum of the maximum of $2 J_{1}$ and the minimum of $J_{z}$. The sharpness of the transition from the launching to the resonance circle is sensitive to the minimum value of $J_{x}$. By using the value of $J_{2}$ obtained by this prescription the minimum value of $J_{z}$ was not overestimated. The value of the constant $\tilde{H}-\left[Q_{30} J_{2}+\alpha_{28} J_{2}^{2} / 2\right]$ was determined by the requirement that $\cos \phi_{1}$ was equal to \pm 1 when $J_{1}$ was a maximum. Whether $\cos \phi_{1}$ was set to +1 or to -1 depended upon which side of the separatrix the particle was on. In Fig. 3 six data sets are shown for which the strength of the vertical kick was held constant while the strength of the horizontal kick was incremented, along with the fits to Eq. 4. For five of the kicks shown in Fig. 3 the measured and predicted tunes are shown in Fig. 4.

\section{Conclusion}

The betation difference resonance, $Q_{z}-2 Q_{z}=-6$, was investigated at the IUCF cooler ring. It was found that a single resonance Hamiltonian accurately described the coupled motion. It was possible to match both the phase advance per turn of $\phi_{1}$ and the contour traced in the map of $\left(\sqrt{J_{1}} \cos \phi_{1}, \sqrt{J_{1}} \sin \phi_{1}\right)$ with the predictions from the Hamiltonian Eq. 4. The values of the resonance strength $g$, and of the tune shift with amplitude parameters, $\alpha_{11}$, and $\alpha_{z x}-\alpha_{z x}$ were determined. The agreement with the predicted values of these quantities is under investigation.

\section{References}

[1] E.D. Courant and H.S. Snyder, Ann. Phys. (NY) vol. 3, p. 1 (1958).

[2] R. Ruth, in Physics of Particle Accelerators, AIP Conf. Proc. No. 153 p. 150 (1987).

[3] S.Y. Lee, et al., Phys. Rev. Lett. 67, 3768 (1991); D.D. Caussyn, et al., Phys. Rev A bf 46, 7942 (1992).

[4] Y. Wang, et al., "Experimental Results of the Sum Resonance", in these proceedings. 